\title{
Platinum Resistance Thermometer with Metrological Self-Check
}

\author{
Yulia Baksheeva, Ksenia Sapozhnikova, Roald Taymanov \\ D.I.Mendeleyev Institute for Metrology (VNIIM), St.Petersburg, Russia \\ baksheyeva@rambler.ru, k.v.s@vniim.ru, taymanov@vniim.ru
}

\section{Introduction}

The need for reliable performance of automatic equipment with embedded sensors including widely-spread temperature sensors, issues the challenge of checking their metrological serviceability automatically.

The similarity of ways the evolution of biological systems and development of technical systems pass, shows that the most promising solution of the problem indicated is the self-check of sensors (metrological self-check) [1-4].

According to [4] the sensors with metrological self-check are called intelligent ones. The metrological self-check can be implemented in two forms: a direct metrological self-check using an embedded measuring instrument of a higher accuracy and metrological diagnostic self-check realized without application of means of such a kind.

The metrological diagnostic self-check is accomplished on the basis of evaluating a deviation of the parameter characterizing a critical uncertainty component from an accepted reference value of this parameter. By the critical uncertainty component they imply a dominating uncertainty component or that tending to rise quickly.

The procedure for developing a sensor able to perform self-checking includes the following metrological investigations:

- $\quad$ analysis of potential sources of uncertainty taking into account technology and design;

- $\quad$ ranking of uncertainty components, which originate in the process of sensor operation, on the basis of the probability to exceed permissible limits;

- $\quad$ selection of a critical uncertainty component;

- detection of redundant measurement information available in a sensor for evaluating a level of the critical uncertainty component.

On the basis of these investigations a sensor structure with the redundancy that is necessary to provide the metrological self-check, is developed.

In the paper the procedure for developing a sensor with metrological self-check is illustrated by the example of a platinum resistance thermometer (PRT).

\section{Dismountless metrological serviceability check of resistance thermometers}

The well-known methods for performing the dismountless check are based on additional information obtained from a sensor or set of sensors included into a multi-channel measuring system.

The check of metrological serviceability of sensors included into a multi-channel measuring system is done in a mode at which the sensors are affected by a medium having a presumably identical temperature [5]. At the same time, either a total average temperature or a temperature value measured with a reference sensor is considered as a conventional true value.

In practice, a serious disadvantage of such an approach is the necessity to equalize a temperature field over the total space where the sensors are located, the uncertainty being less by several times than a permissible uncertainty of measurements. Moreover, the assurance of the special conditions for operating the equipment used requires significant material and time costs.

A set of temperature sensors, the metrological serviceability of which has to be checked on-line, are placed in the equipment close to each other [5]. A weakness of this method as well as of the method mentioned above (in a version without application of a reference sensor) is its inability to detect a systematic uncertainty component caused by a synchronous drift of graduation characteristics of the sensors.

The temperature sensor containing a number of sensitive elements (SE) in one case, in particular two of them [6], does not also detect an uncertainty caused by the synchronous drift.

In some sensors an additional channel is used. This enables a reference thermal sensor to be periodically installed into the channel [7-9]. The main disadvantage of the method is the fact that in practice the checking procedure cannot be frequently repeated. The procedure of such a type is connected with a mechanical effect upon the sensor and can result in either some damage of the 
equipment or an uncertainty increase due to an inaccuracy of reference sensor installation.

To check up the metrological serviceability of temperature sensors, a capsule with a reference material (in combination with a heater or without it) is used [10-12]. The heater and capsule significantly increase a sensor mass making worse its response time. Without any heater the sensor check can be performed only at those preliminary unknown moments of time when the medium temperature to be measured changes and achieves the melting point of the reference material.

The strictest method for checking up the metrological serviceability of autonomous sensors without dismounting them is the method of measuring a thermal noise (Johnson noise) [13, 14]. However, the Johnson noise represents itself as a very weak signal. Therefore, its detection against a background of industrial interferences and noises of a signal transmittance circuit is an extremely complicated task, the solution of which requires significant computational resources and inputs.

The analysis carried out shows that at present the problem of on-line checking the metrological serviceability of resistance temperature sensor has not yet been solved.

\section{Theoretical grounds of the method of metrological diagnostic self-check}

The development of a PRT with the self-check was carried out by the authors on the basis of the well-known "strain free" design suitable for performing experimental researches. At the same time, the opportunities to use some other technologies were taken into account.

The "strain free" design, which is in detail considered in [15], is a ceramic tube with longitudinal holes, where the spirals of platinum wires filled up with ceramic powder are located. The tube is hermetically sealed. Leading-out wires are soldered to the ends of the spirals. The technology of manufacturing the sensors of such a type contains a noticeable part of manual operations. To control the quality of each operation is extremely difficult. The character of the processes generating an increase of the PRT uncertainty, irrespective of the design and technology of manufacturing, significantly changes depending on a working temperature range.

If one sums up the known data [15-20], then within the working temperature range up to $T_{\max }<(0,25-0,30) T_{m}$, where $T_{\max }$ is its upper limit and $T_{m}$ is the melting point of platinum (which corresponds to $T_{\max } \approx(450-500)^{\circ} \mathrm{C}$ ), the main processes affecting the change of the PRT uncertainty in the process of operation can be divided into two main groups.

The first group includes the processes leading to destruction of a thin surface layer of conductors and variation of its conductive properties with regard to all SEs of the sensors:

- $\quad$ surface oxidation;

- $\quad$ sublimation of surface substances;

- $\quad$ contamination of the surface layer by the diffusion of oxides and mixtures from the ceramic fill-up;

- mechanical damage of the surface, and so on.

Under the influence of the uncertainty sources, which refer to the first group, in the course of time the specific resistance of the surface layer begins to exceed significantly the specific resistance of a conductor material. This can be represented as a certain equivalent decrease of an area of the crosssection of the platinum wire, which correspondingly increases its resistance.

Other processes (which are not connected with the destruction of the surface layer) leading to an unexpected change of resistance of some SEs refer to the second group:

- consequences of the technological spoilage, which have not been detected in the production process (a change of contact resistance caused by low-quality welding or soldering, origination of mechanical strains and deformations as a result of heating or cooling of a SE in case of its poor fixation, and so on);

- $\quad$ variation of those parameters of the sensor elements, which are external with respect to the SE spirals (drop of the specific resistance of the sealing layer, increase of its gas- and/or humidity permeability, e.g., due to cracking, etc.).

The influence of the processes, which refer to the second group, on the sensor uncertainty to a great extent depends on the accepted design and technology of manufacturing the PRTs, as well as on the efficiency of the quality control of the production process. When the processes of the first group dominate, a critical uncertainty component is the deviation of the platinum wire resistance, which is caused by variation of the properties of the wire surface layer.

In [21] it is proposed to use the redundancy for realizing the function of metrological diagnostic selfcheck, i.e., to make a SE of two or a number of parts differently sensitive to factors influencing on the growth of the critical uncertainty component.

To realize the function of metrological diagnostic self-check in the process of operation, the diagnostic function (DF) $\beta$ that depends on the values of signals coming from various parts of the SE is calculated. For example, $\beta=R_{1} / R_{2}$. 
At the stage of the original calibration of the temperature sensor, which corresponds to the start of operation, a nominal value $\beta_{0}$ of the DF is determined. A relative deviation $\delta \beta$ of the DF from the nominal value is rigidly connected with the sensor uncertainty. In the process of operation the check of the metrological serviceability is performed by determining $\delta \beta$ at a temperature measured and comparing it with a permissible relative deviation.

When the value $\delta \beta$ exceeds the permissible limits or approaches to such a limit, it is necessary to perform an unscheduled calibration of the sensor even if the specified interval has not come to its end.

When the specified calibration interval comes to the end and $\delta \beta$ does not exceed a permissible value, then this fact can become an argument for significant increasing the corresponding interval and using the sensor further.

In the general case (for various designs of the PRT), it is necessary to provide a different ratio of the cross-section area to the perimeter of the cross-section of the SE parts.

In each part of the SE the destruction rate (rate of resistance variation) of the conductor surface layer weakly depends on the geometrical parameters of the conductors themselves, and the depth of the destructed layer is small as compared to linear dimensions of the conductor cross-section.

Then for the simplest version of the SE including two parts

$$
\delta \beta=\left|\frac{\beta-\beta_{0}}{\beta_{0}}\right|=\left|\frac{\beta}{\beta_{0}}-1\right| \approx\left|\frac{1-a \frac{P_{2}}{S_{2}}}{1-a \frac{P_{1}}{S_{1}}}-1\right|, \text { where }
$$

$a$ is the assumed thickness of the surface layer subjected to the destruction;

$P_{1}, P_{2}$ and $S_{1}, S_{2}$ are the perimeters and areas of the cross-sections of the SE parts having different sensitivity to the factors influencing on the growth of the critical uncertainty component, correspondingly.

Provided the relationship between the $\delta \beta$ value and uncertainty $\delta T$ of the temperature measurement is known, then, using a value of $\delta \beta$ determined experimentally, it is possible to introduce a corresponding correction into a measurement result.

For the "strain free" design it is convenient to manufacture the SE spirals made of the wires of various diameters.

Then

$\delta \beta \approx 4 a\left|\frac{d_{1}-d_{2}}{d_{1} d_{2}}\right|$, where $d_{1}$ and $d_{2}$ are the wire diameters.

\section{Results of experimental investigations}

For preliminary evaluation of the efficiency of the considered metrological self-check method, there were carried out experimental investigations of the SE prototypes. This was done in the mode of a forced load, "heating up to $600^{\circ} \mathrm{C}$ - cooling", with a cycle time of $70-80$ hours. The prototypes contained a SE consisting of two spirals (each having a resistance of $100 \mathrm{Ohm}$ ) which were made of a platinum wire of 50 and $30 \mu \mathrm{m}$ in diameter. In each cycle the resistance of each spiral was measured at temperature of 0,450 and $600{ }^{\circ} \mathrm{C}$ determined with a reference thermometer. Then the values of $\beta, \delta \beta$ and resistance deviation (increment) $\Delta R$ for each spiral were calculated.

Before the experiments a preliminary annealing of the SEs was done at temperature of $800^{\circ} \mathrm{C}$ to stabilize the SE characteristics. After that, they were calibrated with uncertainty of $\pm 0,2^{\circ} \mathrm{C}$.

It was noticed that during the first cycles of the forced load the process of annealing was still in progress, its rate being different due to the fact that cold-hardening of the wires differed in diameter was not the same. Therefore, the characteristics obtained after annealing of both two spirals were taken as the calibration characteristics.

A zero value was assigned to the corresponding cycle; the resistance increments $\Delta R$ for each cycle being determined with respect to the resistance value measured in the zero cycle. On the basis of the data obtained, the DF values $\beta_{0}$ for the zero cycle were calculated, and for the subsequent cycles the DF relative deviation $\delta \beta$ and relative uncertainty $\delta T$ of temperature measurements were determined.

The following results were obtained.

In a majority of the prototypes the resistance of each spiral gradually changed with increasing the number of cycles, a resistance increment $\Delta R$ of the spiral made of the wire of $30 \mu \mathrm{m}$ in diameter being greater than that of the $50 \mu \mathrm{m}$ wire. The relative deviation of the DF proportionally changed with respect to the relative uncertainty of temperature measurement. The characteristic dependencies are illustrated in Fig. $1 \mathrm{a}$ and $1 \mathrm{~b}$. 


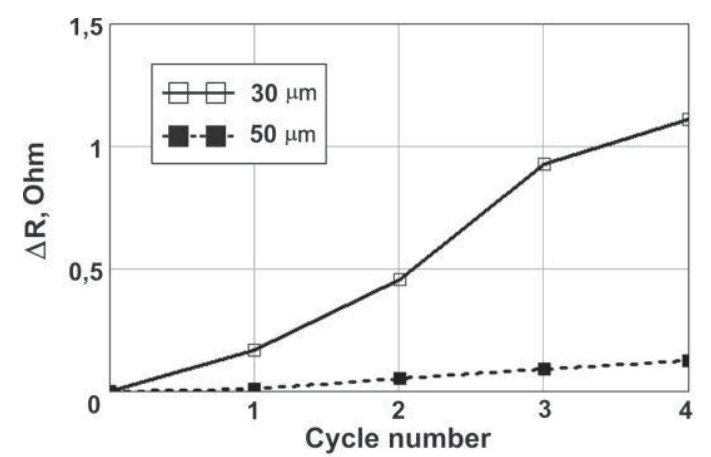

a)

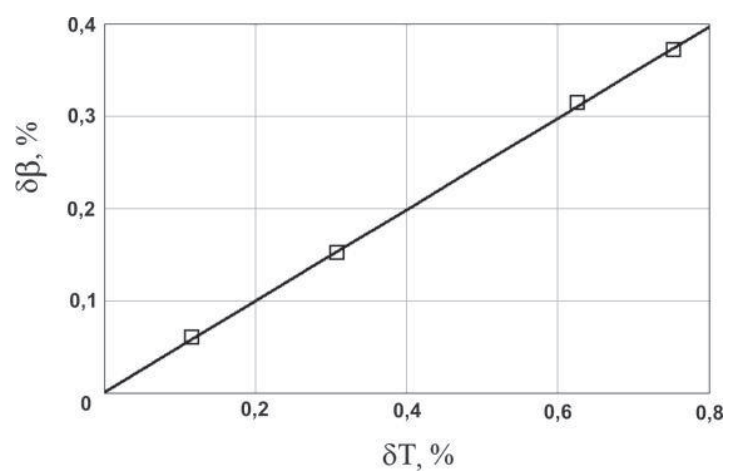

b)

Fig.1. The characteristic dependencies obtained in the experiment at temperature of $450^{\circ} \mathrm{C}$

a) Dependence of the resistance increment in the SE parts on the cycle number;

b) Dependence of the relative DF deviation on the relative uncertainty of temperature measurements

The type of these dependencies confirms that the SEs are predominantly influenced by the processes relating to the first group. However, in some prototypes among those investigated, there have been fixed up metrological defects caused by the processes of the second group.

The experiment performed has shown that the metrological self-check method enables a cumulative uncertainty to be evaluated and metrological defect to be revealed. Moreover, in a number of cases this method makes it possible to determine the source of the defect occurred. However, the development of the sensor with two spirals of the wire of different diameters requires introducing some changes into the design and technology of the sensor.

\section{Resources for improving the sensors}

Taking into account the experimental investigations, it was recognized that the PRT design with the metrological self-check should provide for:

1) assembling SE parts into a single case;

2) providing the closeness of original resistance values of SE parts;

3) manufacturing SE parts with maximally identical design parameters, i.e., with close values of a pitch, length and diameter of spirals.

Conditions 2) and 3) can be jointly satisfied if the first SE part is made of a single conductor and the second part contains several conductors connected in parallel.

At the same time, the length values of the conductors of both SE parts should be close to each other. The similar condition is required for the relationship between the cross-section area of a single conductor (a thicker one) of the first SE part and total cross-section area of the second part conductors [22].

Fig. 2 illustrates the dependence between a complex parameter $\sigma$ characterizing the total effect of the $\delta \beta$ maximum value and maximum identity of the SE parts, and relationship $b=d_{1} / d_{2}$ of the diameters of both parts of the SE. The optimal number of conductors in the SE part made of a thinner wire is 2 . The choice of wire diameters is limited by a standard gauge of the wire produced by industry. For example, the spiral pairs made of wire of $80 \mu \mathrm{m}$ and $56 \mu \mathrm{m}(b \approx 1,43), 70 \mu \mathrm{m}$ and $50 \mu \mathrm{m}(b \approx 1,41), 100 \mu \mathrm{m}$ and $70 \mu \mathrm{m}(b \approx 1,43)$ can be applied.

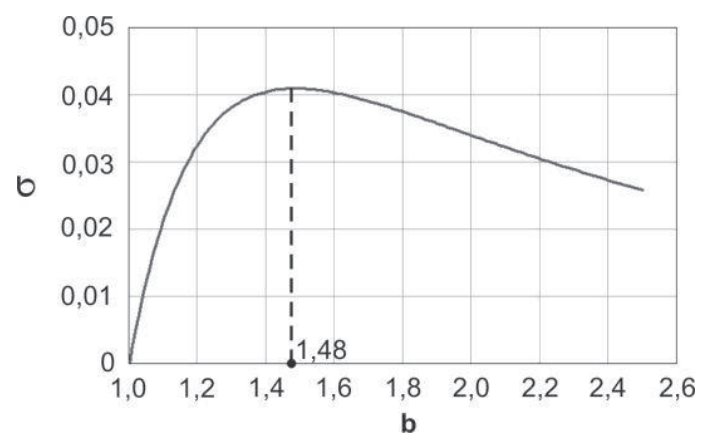

Fig.2. Dependence between the complex parameter $\sigma$ and relationship of the wire diameters 
In manufacturing the SE parts on the basis of the "strain free" design as well as in applying such a sensor, some difficulties connected with an insufficient mechanical strength and rigidity of the spiral made of thin wires can arise.

To remove this disadvantage it is useful to make the second SE part of twisted wires connected in parallel. The mechanical strength and rigidity of the spiral made of the twisted wires is comparable with the corresponding parameters of the first part mentioned above. This increases the stability and significantly simplifies the technology of manufacturing. The possibility of contact of the wire pieces in the unit of such a type will not result in a noticeable uncertainty since the closely located points of two conductors are characterized by approximately equal potential.

\section{Conclusion}

An intelligent temperature sensor with metrological self-check can be implemented on the basis of the technologies applied in industry. Its advantages are:

- $\quad$ automatic check of the measurement information reliability in the process of operation;

- $\quad$ opportunity of automatic correction of uncertainty;

- availability of diagnostic information that allows the calibration to be realized according to a technical condition;

- $\quad$ possibility to increase by many times the calibration interval (as compared to a sensor of the similar type, but without the metrological diagnostic self-check).

\section{References}

1. Yu. Tarbeyev, A. Kuzin, R. Taymanov, A. Lukashev, New Stage in the Metrological Provision for Sensors, Measurement Techniques, vol. 50, No 3, 2007, pp. 344-349.

2. R. Taymanov, K. Sapozhnikova, Intelligent Measuring Instruments. Maximum Reliability of Measuring Information, Minimum Metrological Maintenance, Proc. of the XVII IMEKO World Congress, Dubrovnik, Croatia, June 22-27 2003, pp. 1094-1097, CD-ROM. Online: http://www.imeko.org/

3. R. Taymanov, K. Sapozhnikova, Metrological Self-Check and Evolution of Metrology, Measurement, vol. 43, No 7, 2010, pp. 869-877.

4. GOST R 8.673-2009. Intelligent Sensors and Intelligent Measuring Systems. Basic Terms and Definitions. (in Russian).

5. H. M. Hashemian, Sensor Performance and Reliability. USA: ISA, 2005.

6. http://www.omega.com/pptst/TC-K-NPT_RTD-NPT.html

7. RU2299408 G01K 7/02, G01K 13/12, G01K 15/00, 20.05.2007, Bul. No 14, A. V. Karzhavin, V. A. Karzhavin, V. V. Bogatov, A. V. Belevtsev, Device for Measuring Temperature in Form of Thermoelectric Transformer.

8. http://www.tesey.com

9. http://npk-etalon.ru/

10. A. A. Sachenko, Embedded Instruments for Metrological Check and Correction of Errors in Measuring Channels, Investigations in the Field of Metrology for Automated Systems of Control of Technological Processes, Lvov, VNIIMIUS, 1986, pp. 74-78. (in Russian).

11. F. Bernhard, D. Boguhn, S. Augustin, H. Mammen, and A. Donin, Application of Self-calibrating Thermocouples with Miniature Fixed-point Cells in a Temperature Range from $500^{\circ} \mathrm{C}$ to $650^{\circ} \mathrm{C}$ in Steam Generators, Proc. of the XVII IMEKO World Congress, Dubrovnik, June 22-27 2003, pp. 1604-1608, CDROM. Online: http://www.imeko.org/

12. http://www.electrotherm.de

13. B. N. Oleynik, V. M. Sumerin, et al., Temperature Measurement Using Thermonoise Thermometers, Measurements, Control, Automation, vol. 45, No 1, 1983, pp. 22-29. (in Russian)

14. B. Stadnyk, I. Mykytyn, Mathematical Models of Methodical Error for Noise Thermometers, Sensors \& Transducers, vol.123, No 12, 2010, pp. 76-83.

15. G. V. Samsonov, A. I. Kitz, O. A. Kuzenia, V. I. Lakh, et al., Sensors for Measuring Temperature in Industry, Kyev, Naukova Dumka, 1972. (in Russian).

16. R. J. Berry, Effect of Pt Oxidation on Pt Resistance Thermometry, Metrologia, vol. 16, 1980. p.117.

17. R. J. Berry, Oxidation, Stability and Insulation Characteristics of Rosemount Standard Platinum Resistance Thermometers, Temperature, Its Measurement and Control in Science and Industry, New York, AIP, vol. 5, 1982, pp. 753-761.

18. L. Crovini, A. Actis, G. Coggiola, A. Mangano, Precision Calibration of Industrial Platinum Resistance Thermometers, Temperature: Its Measurement and Control in Science and Industry, New York, AIP, vol. 6, 1992, pp. 1077-1082. 
19. H. M. Hashemian, K. M. Petersen, Achievable Accuracy and Stability of Industrial RTDs, Temperature: Its Measurement and Control in Science and Industry, New York, AIP, vol.6, 1992, pp. 427432.

20. X. Li, M. Zhao, and D. Chen, A Study on the Stability of Standard Platinum Resistance Thermometer in the Temperature Range from $0^{\circ} \mathrm{C}$ through $720^{\circ} \mathrm{C}$. http://www. hartscientific.com

21. RU2321829 G01 D 3/00, 10.04.2008, Bul. No 10, L. P. Gorokhov, K. V. Sapozhnikova and R. E. Taymanov, Mode of Control of Metrological Condition of Measuring Converter of Non-electric Value and Installation for its Execution.

22. RU2010142833 G01D 3/00, 07.10.2010, Yu.V. Baksheeva, K. V. Sapozhnikova, R. E. Taymanov, Temperature Measuring Transducer with Check of Metrological Serviceability. 